\title{
Cirugía endoscópica nasal en el tratamiento de papiloma invertido: A propósito de 18 casos
}

\author{
Endoscopic sinus surgery in the management of inverted papilloma: \\ An analysis of 18 cases
}

\author{
Sofía Waissbluth $A^{1,3}$, Karen García $C^{2}$, César Imarai $B^{1,3}$.
}

\begin{abstract}
RESUMEN
Introducción: El papiloma invertido nasosinusal es un tumor benigno con tendencia a la recidiva, y con riesgo de malignización. El tratamiento habitual es la extirpación quirúrgica.

objetivo: Describir y analizar las características clínicas, epidemiológicas, y resultados del tratamiento quirúrgico por vía endoscópica.

Material y método: Estudio retrospectivo, descriptivo, incluyendo pacientes con papiloma invertido nasosinusal sometidos a extirpación quirúrgica endoscópica entre los años 2012 y 2016. Se analizaron variables epidemiológicas, clínicas, complicaciones quirúrgicas, recurrencias y transformación maligna. El grado de extensión se evaluó con tomografía computarizada según la clasificación de Krouse.

Resultados: La serie está constituida por 18 pacientes, con una edad promedio de 57,4 años. La clínica se caracterizó por obstrucción nasal unilateral 72,2\% (13/18 pacientes), rinorrea $33,3 \%$ (6/18 pacientes), hiposmia $27,8 \%$ (5/18 pacientes) y epistaxis $27,8 \%$ (5/18 pacientes). Según la clasificación de Krouse, las lesiones iniciales se describen como estadio T1 en 7/18, T2 en 5/18, T3 en 5/18 y T4 en 1/18 pacientes. En el período de seguimiento, se observa recurrencia en 4 pacientes en un período promedio de seguimiento de 5,6 meses, y un caso de transformación maligna. No hubo complicaciones quirúrgicas, excepto un caso de epífora por obstrucción de la vía nasolagrimal.

Conclusiones: El abordaje endoscópico fue efectivo para el tratamiento de papilomas invertidos nasosinusales.
\end{abstract}

Palabras clave: Papiloma invertido, Schneideriano, cirugía, endoscopia, recurrencia.

\section{ABSTRACT}

Introduction: Sinonasal inverted papillomas are benign tumors with a tendency to recur and a potential for malignant transformation. Surgical resection is the treatment of choice.

Aim: To describe and analyze the clinical characteristics, epidemiology, and surgical results from an endoscopic endonasal approach.

\footnotetext{
Médico del Departamento de Otorrinolaringología, Pontificia Universidad Católica de Chile.

Médico del Departamento de Otorrinolaringología, Hospital Clínico Universidad de Chile.

Médico del Departamento de Otorrinolaringología, Complejo Asistencial Dr. Sótero del Río.
} 
Material and method: Retrospective and descriptive study of all patients who underwent endoscopic sinus surgery for the treatment of a sinonasal inverted papilloma between the years 2012 and 2016. Variables analyzed include demographics, signs and symptoms, surgical complications, recurrence rate and malignant transformation. Tumor extension was evaluated with the Krouse staging system on computed tomography imaging.

Results: This series includes 18 patients, with a mean age of 57.4 years. Clinically, patients presented with unilateral nasal obstruction $72.2 \%$ (13/18 patients), followed by rhinorrhea $33.3 \%$ (6/18 patients), hyposmia $27.8 \%$ (5/18 patients) and epistaxis $27.8 \%$ (5/18 patients). Based on the Krouse staging system, 7/18 were T1, 5/18 were T2, 5/18 were T3 and 1/18 was T4 on initial evaluation. On follow-up, 4 patients presented with a recurrence at a mean period of 5.6 months, and one patient exhibited malignant transformation. No surgical complications were observed except for one case of epyphora as a consequence of nasolacrimal obstruction.

Conclusions: The endoscopic approach was effective for the treatment of sinonasal inverted papillomas.

Key words: Inverted papilloma, Schneiderian, surgery, endoscopy, recurrence.

\section{INTRODUCCION}

Los papilomas invertidos son tumores benignos de origen epitelial de la vía nasosinusal. Histológicamente, se describe un crecimiento endofítico y una invasión de la superficie epitelial en el tejido conectivo lo cual le da la característica morfológica invertida al tumor ${ }^{1}$. Provendría del ectodermo, a partir de la membrana schneideriana, la cual daría origen a los tres tipos de papilomas: invertidos, evertidos o exofíticos (fungiforme) y de células cilíndricas u oncocítico². Los papilomas schneiderianos representan aproximadamente un $0,4 \%-4,7 \%$ de los tumores nasales y suelen ser unilaterales ${ }^{3}$.

Los papilomas invertidos son tumores infrecuentes con tendencia a la recidiva y con riesgo de malignización. Los sitios más frecuentemente afectados son la pared nasal lateral, el seno maxilar y el etmoides ${ }^{4}$. El tratamiento es quirúrgico para lograr la resección completa de la lesión. La radioterapia se puede considerar en casos de malignización 0 en situaciones en las que no se puede operar. Las recurrencias suelen ocurrir precozmente, en los dos primeros años del tratamiento, aunque se han descrito casos de recurrencia hasta 10 años o más, luego de una intervención quirúrgica5. El tumor recurre principalmente en el sitio inicial de la lesión, lo cual sugiere una resección incompleta como causal de la recidiva ${ }^{6}$. Las tasas de recurrencia descritas en la literatura varían considerablemente según el tipo de cirugía, el estadio del tumor y el tiempo de seguimiento. En general, se describe una recurrencia entre 14\%-25\% $\%^{5,7}$.

Se realizó un metaanálisis de 29 estudios con un total de 3.177 pacientes con un diagnóstico de papiloma schneideriano, y a partir de estos casos, se describe un riesgo de malignización de $9 \%{ }^{8}$. En una revisión extensa realizada en 2009 , se demostró una tasa de malignización de $7 \%$; de un total de 200 pacientes con el diagnóstico de papiloma invertido, 14 presentaron malignización. El tiempo mediano de seguimiento fue de 4,6 años, con un rango de 9 meses a 19 años ${ }^{5}$. Otra revisión de la literatura, evaluando 63 series de casos de papilomas invertidos, describe una incidencia de $7,1 \%$ de carcinoma sincrónico y, 3,6\% de carcinoma metacrónico ${ }^{9}$.

Por el riesgo de recurrencia y malignización, la resección quirúrgica completa es de importancia fundamental. El abordaje externo a través de una maxilectomía medial vía una rinotomía lateral fue el patrón de oro para el tratamiento del papiloma invertido, ya que se logra una exposición amplia de la pared nasal lateral, de la pared medial del maxilar y del etmoides, zonas anatómicas frecuentemente afectadas por este tumor. La técnica de degloving mediofacial es igualmente una buena opción, ya que provee una amplia exposición bilateral y evita una cicatriz facial. Con el avance de las técnicas endoscópicas, se inicia el estudio de la eficacia y seguridad de un abordaje endoscópico transnasal 
para la resección de los papilomas invertidos. Los resultados presentados han sido alentadores, aunque se debe tomar en cuenta el estadio o nivel de severidad de cada caso $0^{10,11}$.

\section{OBJETIVO}

Describir y analizar las características clínicas, epidemiológicas, y los resultados del tratamiento quirúrgico por vía endoscópica, para el manejo de los papilomas invertidos nasosinusales.

\section{MATERIAL Y MÉTODO}

Se realizó un estudio retrospectivo, descriptivo, de todos los pacientes con papiloma invertido nasosinusal sometidos a extirpación quirúrgica primaria por abordaje endoscópico entre los años 2012 y 2016 en el Complejo Asistencial Dr. Sótero del Río. Se revisaron datos epidemiológicos, manifestaciones clínicas, complicaciones quirúrgicas, tasa de recurrencia y de transformación maligna. La técnica quirúrgica fue endoscópica en todos los casos y se realizó con el uso de endoscopios de $0^{\circ}, 30^{\circ}$ y $45^{\circ}$ grados. No hubo casos de abordajes combinados ni abiertos. El sitio de implantación se manejó con la resección del mucoperiósteo y luego fresado de esa zona.

El grado de extensión de la lesión se evaluó con tomografía axial computarizada (TC) de las cavidades paranasales y los hallazgos fueron descritos según la clasificación de Krouse. Esta clasificación fue elaborada y publicada por Krouse con el objetivo de poder comparar los resultados quirúrgicos descritos en la literatura ${ }^{12}$. Se describe de la siguiente forma:

- Estadio T1: Tumor limitado a la cavidad nasal, sin extensión hacia los senos paranasales u espacios extranasales. Sin malignización concomitante.

- Estadio T2: Tumor que involucra el complejo osteomeatal y el etmoides, y/o la porción medial del seno maxilar, con o sin involucrar la cavidad nasal. Sin malignización concomitante.

- Estadio T3: Tumor que involucra la pared lateral, inferior, superior, anterior o posterior del seno maxilar, el esfenoides $y / 0$ el seno frontal, con o sin involucrar la pared medial del seno maxilar, el etmoides o la cavidad nasal. Sin malignización concomitante.

- Estadio T4: Todo tumor con extensión extranasal/extrasinusal que involucre estructuras adyacentes, contiguas como la órbita, el compartimiento intracraneal 0 el espacio pterigomaxilar. Casos de malignización.

\section{RESULTADOS}

\section{Demográfica}

La serie está constituida por 18 pacientes, con una edad promedio de 57,4 años (rango 39-69 años); 10 hombres y 8 mujeres. Siete pacientes presentan hábito tabáquico. El período de seguimiento fue de 2,3 años en promedio (rango: 1 mes-5 años). Uno de los pacientes presenta el antecedente de la tríada de Samter.

\section{Manifestaciones clínicas}

La sintomatología más frecuente fue la obstrucción nasal unilateral $(72,2 \% ; 13 / 18$ pacientes) seguida de rinorrea anterior (33,3\%; $6 / 18$ pacientes), hiposmia $(27,8 \% ; 5 / 18$ pacientes) y episodios de epistaxis unilaterales $(27,8 \%$; $5 / 18$ pacientes). Menos frecuente fueron la descarga posterior $(16,7 \% ; 3 / 18$ pacientes), y algia facial $(16,7 \%$; $3 / 18$ pacientes). Un paciente describe sensación de pesadez facial luego de un caso de recurrencia.

\section{Extensión del tumor}

Según la clasificación de Krouse previamente descrita, se observa que la mayoría de las lesiones se presentaron inicialmente en estadio T1 y T2. El más infrecuente fue el estadio T4, es decir el estadio más avanzado y grave (Figura 1). Un caso se presentó en estadio T4 ya que se observó compromiso de la órbita en el TC de las cavidades paranasales.

En el período de seguimiento, se observa recurrencia en 4/18 pacientes en un tiempo promedio de seguimiento de 5,6 meses. De estas recurrencias, 1 paciente presentó transformación maligna. El grado de Krouse de los casos recurrentes al 


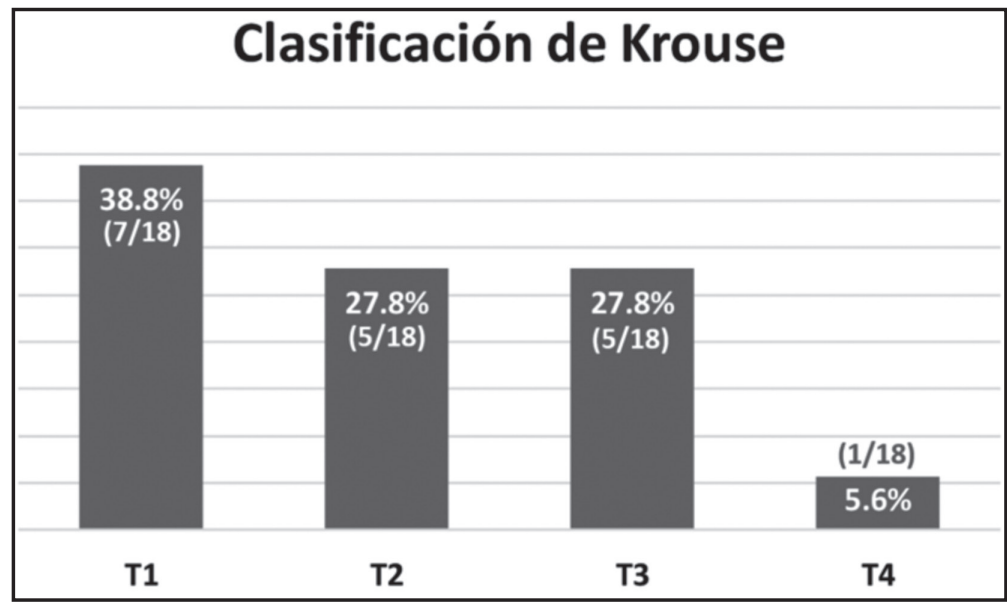

Figura 1. Distribución de los casos según la clasificación de Krouse para la extensión de las lesiones. En los casos de recurrencias, se describe el estadio inicial de la primera cirugía.

momento de la primera cirugía fue T1 en 3 de los 4 pacientes, y T3 en un paciente. Los pacientes que presentaron recidiva fueron operados nuevamente con técnica quirúrgica endoscópica y el paciente con carcinoma recibió además tratamiento con radioterapia.

En la Figura 2, vemos un ejemplo de un paciente que presentó un tumor estadio T3. Luego de 13 meses, presenta una recurrencia con transforma- ción maligna, con diagnóstico anatomopatológico de carcinoma escamoso infiltrante moderadamente diferenciado.

\section{Complicaciones quirúrgicas}

En esta serie, no hubo complicaciones como fístula de líquido cefalorraquídeo, alteraciones oculares ni olfatorias. Hubo un caso de epistaxis

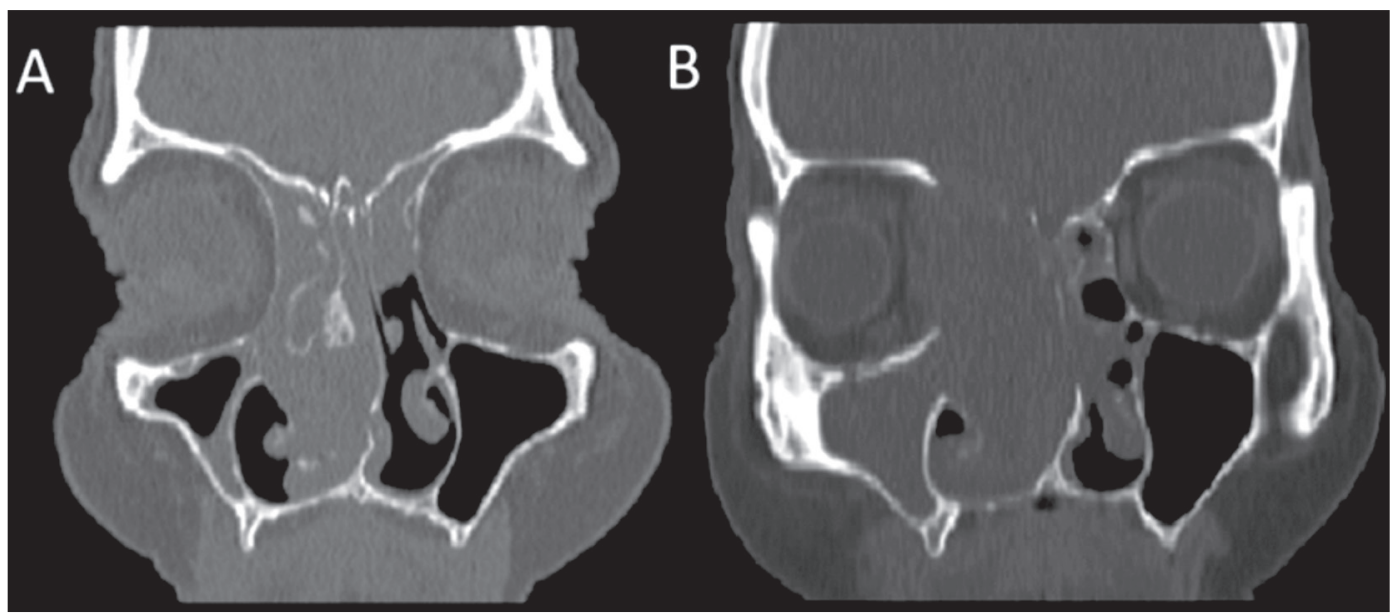

Figura 2. Tomografía computarizada (TC) de las cavidades paranasales. Caso de transformación maligna. (A) TC inicial que muestra compromiso de la pared medial del seno maxilar, el receso frontal, el etmoides y la cavidad nasal derecha. (B) TC de recurrencia con lesión que compromete la cavidad nasal, el etmoides y el seno maxilar derecho con invasión a la órbita y fosa craneal anterior. 
en el primer control posoperatorio, tras el retiro del taponamiento nasal colocado durante la cirugía. El caso se resuelve con taponamiento anterior unilateral por 5 días. Hubo igualmente un caso de epífora ipsilateral por obstrucción de la vía nasolagrimal posquirúrgica. Resulta importante destacar, que este último caso fue durante la cirugía de un paciente con recurrencia y transformación maligna.

\section{DISCUSION}

El papiloma invertido forma parte de la clasificación de los papilomas schneiderianos que además incluye el papiloma oncocítico (de células cilíndricas) y el papiloma exofítico (fungiforme). El más frecuente es el papiloma invertido y tiende a insertarse en la pared nasal lateral, el seno maxilar y el seno etmoidal ${ }^{2,13}$. Es más frecuente en el sexo masculino con una relación de hombre a mujer de $3: 1$, y tiende a presentarse en edad media, más frecuentemente entre los 50 y 60 años ${ }^{5}$. En nuestra serie, la edad promedio fue de 57,4 años, pero la relación de hombre a mujer fue de 1,25:1. Puede presentarse de forma simultánea a una rinosinusitis crónica y esta asociación es igualmente más frecuente con el subtipo de papilomas invertidos, uno de los pacientes de la serie presentó este diagnóstico asociado ${ }^{13}$.

El diagnóstico de esta patología es de importancia, ya que su resección incompleta puede llevar a una recurrencia, la cual se estima entre $14 \%$ y $25 \%$ de los casos, y por su potencial de malignización ${ }^{5,7}$. La probabilidad de recurrencia depende de la técnica quirúrgica, tamaño del tumor y sitio de inserción. En este caso, 4 pacientes $(22,2 \%)$ presentaron una recurrencia en un tiempo promedio de seguimiento de 5,6 meses, lo cual es concordante con la literatura actual, ya que tiende a ocurrir en los primeros 2 años posoperatorios. Además, el hábito tabáquico ha sido considerado como factor de riesgo para la recurrencia de un papiloma invertido ${ }^{14}$. En esta serie, solo un paciente que presentó una recurrencia relata el antecedente de hábito tabáquico, y dos pacientes presentan displasia en el estudio histopatológico, aunque según Mirza y cols (2007) no existiría una asociación entre displasia y riesgo de recurrencia 0 de malignización ${ }^{9}$, por lo cual estos casos se podrían tratar de la misma forma que un papiloma invertido benigno. Sin embargo, la literatura evaluada por estos autores presenta limitaciones, ya que no todas las series reportan la presencia 0 ausencia de displasia?.

Kim y cols (2017) realizaron un metaanálisis para evaluar riesgo de recurrencia según el abordaje quirúrgico. Demostraron un beneficio para el abordaje endoscópico transnasal en los estadios menos avanzados con una reducción del riesgo de recurrencia en $45 \%$ para el estadio $\mathrm{T} 1$ de Krouse, no así para los casos invasivos ${ }^{15}$. La clasificación inicial de Krouse de los casos recurrentes en nuestra serie fue T1 en 3 pacientes, y T3 en un paciente. Aun con un estadio poco avanzado, puede ocurrir recurrencia y depende en parte del sitio de inserción del tumor.

Con el avance de los endoscopios, la visualización directa de la lesión y de su sitio de inserción, ha mejorado la eficacia y seguridad de las cirugías endoscópicas. Diversos estudios han comparado los resultados de los abordajes endoscópicos y abiertos para el manejo de los papilomas invertidos. Una revisión exhaustiva de la literatura realizada por Busquets y Hwang (2006), describe un porcentaje de recurrencia de $12 \%$ en los pacientes operados vía endoscópica, y de $20 \%$ para los abordajes abiertos ${ }^{16}$. Se incluye en el grupo endoscópico, terapias coadyuvantes como la antrostomía maxilar o abordaje tipo Caldwell-Luc. Aunque se escribe un porcentaje menor de recurrencia en el grupo tratado vía endoscópica, se debe tener en consideración, que los casos más avanzados, con malignización y/o con sitios de inserción laterales en las cavidades, difícilmente serán operados únicamente por vía endoscópica ${ }^{4,16}$.

\section{CONCLUSION}

Según el estadio del tumor y experiencia del cirujano, se puede considerar el abordaje endoscópico transnasal para el manejo de los papilomas invertidos con buenos resultados funcionales y pocas complicaciones. Es importante recalcar la necesidad de un seguimiento a largo plazo dado el riesgo de recurrencia y malignización 
de los papilomas. Aun en los casos con estadio poco avanzado inicialmente, el riesgo de recurrencia existe. El riesgo de malignización en esta serie fue

\section{BIBLIOGRAFIA}

1. VRABEC DP. The inverted Schneiderian papilloma: a clinical and pathological study. Laryngoscope 1975; 85: 186-220.

2. Hyams VJ. Papillomas of the nasal cavity and paranasal sinuses. A clinicopathological study of 315 cases. Ann Otol Rhinol Laryngol 1971; 80: 192-206.

3. Barnes L, Eveson J, Reichart P, Sidransky D. Schneidarian papillomas in World Health Organization Classification of Tumours. Pathology and genetics of head and neck tumours. Lyon: IARC Press 2005; 28-33.

4. Krouse JH. Endoscopic treatment of inverted papilloma: safety and efficacy. Am J Otolaryngol 2001; 22: 87-99.

5. Lawson W, Patel ZM. The evolution of management for inverted papilloma: an analysis of 200 cases. Otolaryngol Head Neck Surg 2009; 140: 330-5.

6. Anari S, Carrie S. Sinonasal inverted papilloma: narrative review. J Laryngol Otol 2010; 124: 705-15.

7. Katori H, Nozawa A, Tsukuda M. Histopathological parameters of recurrence and malignant transformation in sinonasal inverted papilloma. Acta Otolaryngol 2006; 126: 214-8.

8. Re M, GioacchinI FM, Bajraktari A, et al. Malignant transformation of sinonasal inverted papilloma and related genetic alterations: a systematic de $5,6 \%$, por lo tanto, se debe siempre considerar esta posibilidad cuando enfrentamos pacientes con papilomas invertidos nasosinusales.

review. Eur Arch Otorhinolaryngol 2017; 274 : 2991-3000.

9. Mirza S, Bradley PJ, Acharya A, Stacey M, Jones NS. Sinonasal inverted papillomas: recurrence, and synchronous and metachronous malignancy. J Laryngol Otol 2007; 121: 857-64.

10. Sauter A, Matharu R, Hörmann K, Naim R. Current advances in the basic research and clinical management of sinonasal inverted papilloma (review). Oncol Rep 2007; 17: 495-504.

11. Reh DD, Lane AP. The role of endoscopic sinus surgery in the management of sinonasal inverted papilloma. Curr Opin Otolaryngol Head Neck Surg 2009; 17: 6-10.

12. KROUSE JH. Development of a staging system for inverted papilloma. Laryngoscope 2000; 110: 965-8.

13. Vorasubin N, Vira D, Suh JD, Bhuta S, Wang MB. Schneiderian papillomas: comparative review of exophytic, oncocytic, and inverted types. Am J Rhinol Allergy 2013; 27: 287-92.

14. Rон HJ, Mun SJ, Chо KS, Hong SL. Smoking, not human papilloma virus infection, is a risk factor for recurrence of sinonasal inverted papilloma. Am J Rhinol Allergy 2016; 30: 79-82.

15. KIM JS, Kwon SH. Recurrence of sinonasal inverted papilloma following surgical approach: A meta-analysis. Laryngoscope 2017; 127: 52-8.

16. Busquets JM, Hwang PH. Endoscopic resection of sinonasal inverted papilloma: a meta-analysis. Otolaryngol Head Neck Surg 2006; 134: 476-82. 